\title{
Lagerung und Abdeckung von Beckenfrakturen
}

Alexander Motzny, Tobias Maximilian Kraus, Armin Mees

\section{Einleitung}

Die Einteilung und die Klassifikation der Becken- und Azetabulumfrakturen gestaltet sich selbst für den Geübten als komplexe Angelegenheit. Es resultieren aus der Frakturklassifikation und -morphologie multiple Zugangswege und Operationstechniken. Dies wiederum hat unzählige differenzierte Lagerungsmöglichkeiten zur Folge.

Im Vordergrund für die Versorgung von Beckenring- und Azetabulumfrakturen stehen die Rückenlage, die Seitenlage und die Bauchlage. Unser Augenmerk soll hauptsächlich auf der Rücken- und Seitlagerung liegen, jedoch wollen wir die Bauchlagerung auch kurz ansprechen. Natürlich existieren frakturbedingt vielfache Modifikationen und Kombinationen der einzelnen Lagerungstypen.

Besonders wichtig ist die präoperative OP-Tisch-Auswahl und -Vorbereitung. Hier spielt die Röntgendurchlässigkeit des OP-Tisches eine zentrale Rolle.

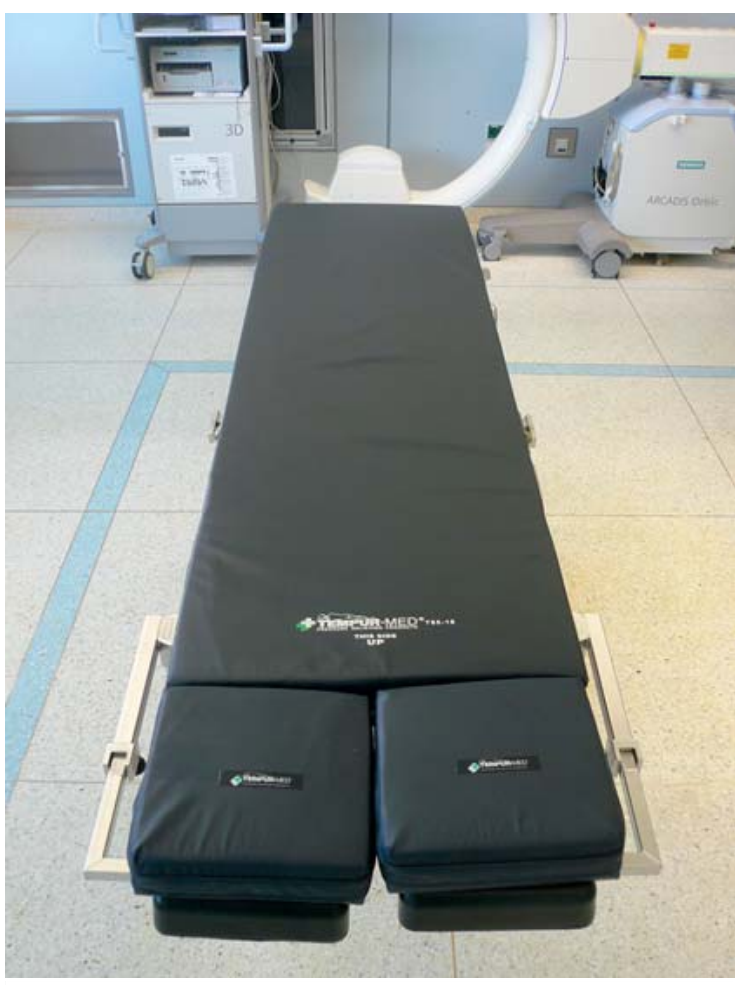

Abb. 1 Standard-OP-Tisch
In der weiteren Abfolge sind natürlich auch das Wärmemanagement und das Vermeiden von Lagerungsschäden von zentraler Bedeutung. Besonderheiten des oft polytraumatisierten Patienten und die damit verbundene Komplexität der „Kollateralverletzungen“ stellen uns oft vor zusätzliche Herausforderungen, mit denen wir nicht gerechnet haben, die wir aber beherrschen müssen. Lagerungshilfsmittel wollen wohl ausgesucht sein und sollten immer in ausreichender Anzahl zur Verfügung stehen.

Nach Klassifikation der Fraktur und Festlegung der operativen Vorgehensweise und damit Festlegung des Zugangsweges erfolgt die Festlegung der Lagerung, des OP-Tisches und der intraoperativ angewandten Röntgenverfahren, die wiederum Auswirkungen auf die Lagerung und unser Setup im OP-Saal haben.

\section{OP-Tisch-Auswahl}

In der Vergangenheit wurden Standard-OP-Tische mit Tisch-/Beinverlängerungen verwendet ( $\bullet$ Abb. 1). Diese sind nun heute im Zeitalter moderner Werkstoffe durch Carbon-OP-Tische ( $\bullet$ Abb. 2 ) abgelöst worden. CarbonOP-Tisch-Verlängerungen oder Tischanbauten aus Carbon mit einer zusätzlichen Stütze stellen eine gute Alternative dazu dar.

Bestimmte Röntgenverfahren, wie das intraoperative CT z. B. mittels eines AIRO (Mobiles CT), benötigen ein besonderes Setup, da Carbon-OP-Tisch, die Gantry und die OP-Tisch-Säule eine Einheit bilden. Dies muss bei der

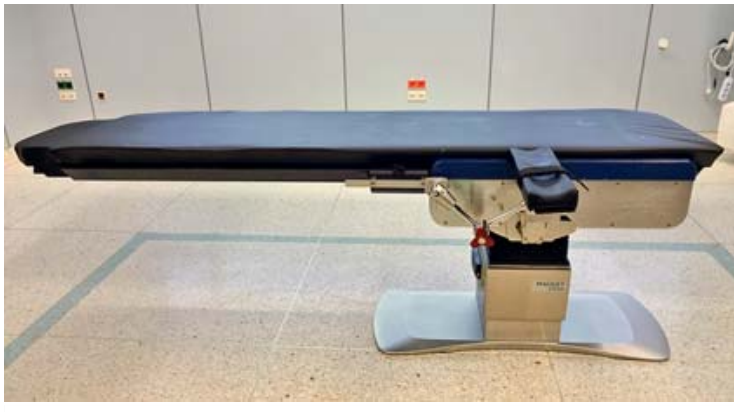

Abb. 2 Carbon-OP-Tisch 
präoperativen Vorbereitung berücksichtigt werden ( $\triangleright$ Abb. 3).

Teilweise existieren Sonder-OP-Tische mit einem sehr begrenzten Einsatzgebiet (Carbonteil für ISG-Verschraubung [ISG: Iliosakralgelenk], > Abb. 4).

Grundsätzlich sollte im Bereich des OP-Gebietes am OPTisch so wenig Metall wie möglich vorhanden sein, da dies einerseits die Durchleuchtung stören kann und andererseits bei 3-D- oder CT-Scans zu einer erhöhten Strahlenbelastung führt und die Bildqualität vermindert. Moderne Bildverarbeitung ist jedoch in der Lage, diese Störungen herauszurechnen.

\section{OP-Tisch-Aufbau und Vorbereitung}

Wir verwenden grundsätzlich für Beckenfrakturen einen großen Carbon-OP-Tisch (Breite $60 \mathrm{~cm}$ ) in umgedrehter Version. Somit können wir eine Verlängerung am Kopfteil anbringen, die den OP-Tisch um knapp $20 \mathrm{~cm}$ verlängert. Sofern man dann noch die C-Bücke zur Befestigung von Anbauteilen vor der OP-Tisch-Säule verbaut, hat man bei voll ausgefahrenem OP-Tisch fast $1,8 \mathrm{~m}$ frei durchleuchtbare Strecke ( $\triangleright$ Abb. 5).

Moderne OP-Tische verfügen heute über eine Weichschaumauflage, die Lagerungsschäden durch langes Liegen und narkosebedingte Bewegungslosigkeit und verminderten Muskeltonus vermeiden soll.

Auf diese OP-Tisch-Lagerungsfläche legen wir ein dünnes, patientenfern gummiertes Zelluloseabdecktuch. Dieses besitzt eine bestimmte, jedoch nur geringe Saugfähigkeit. Unter den Schwerpunkt des Patienten, also im Bereich zwischen Schulter und Gesäß, legen wir eine Inkontinenzunterlage (Safetex, $85 \times 90 \mathrm{~cm}$ ), die zusätzlich eine bestimmte Saugleistung hat. In erster Linie dient sie aber dem scherkräftefreien Transfer des Patienten auf dem OP-Tisch ( $\bullet$ Abb. 6).

Als Anbauten sind standardmäßig eine Armschiene mit Kugelgelenk und Armhalteband und eine Armschlinge ( $\bullet$ Abb. 7) und ein Narkosebügel am OP-Tisch montiert. Ebenso ist ein abwaschbares Kopfkissen und/oder ein Weichschaumkopfring sowie ein Patientensicherheitsgurt mit Polsterung vorhanden.

Das Verwenden einer Röntgenmatte ist kontraindiziert. Deshalb ist der Carbon-OP-Tisch der einzige OP-Tisch in unserer OP-Abteilung, der über keine Strahlenschutzmatte verfügt.

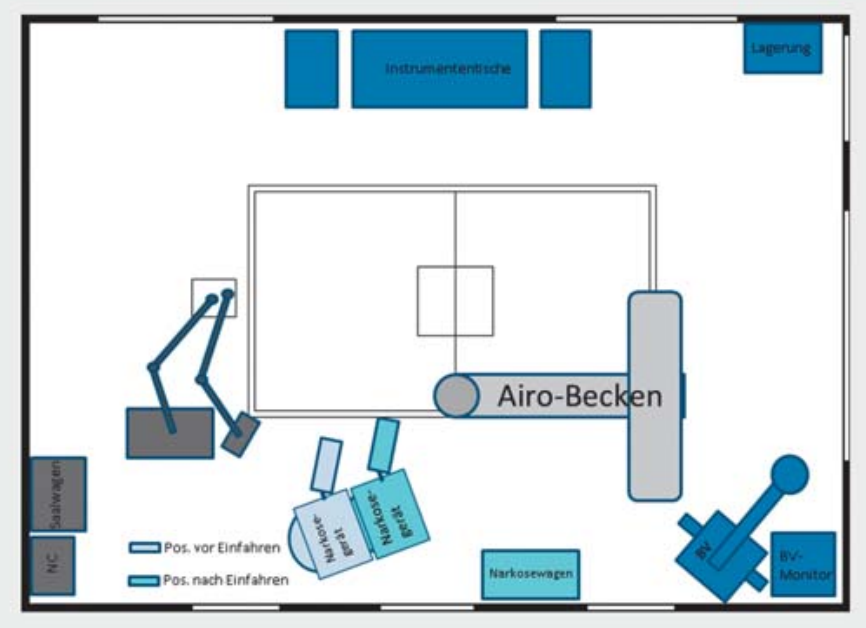

- Abb. 3 Setup Verwendung Mobiles CT im OP

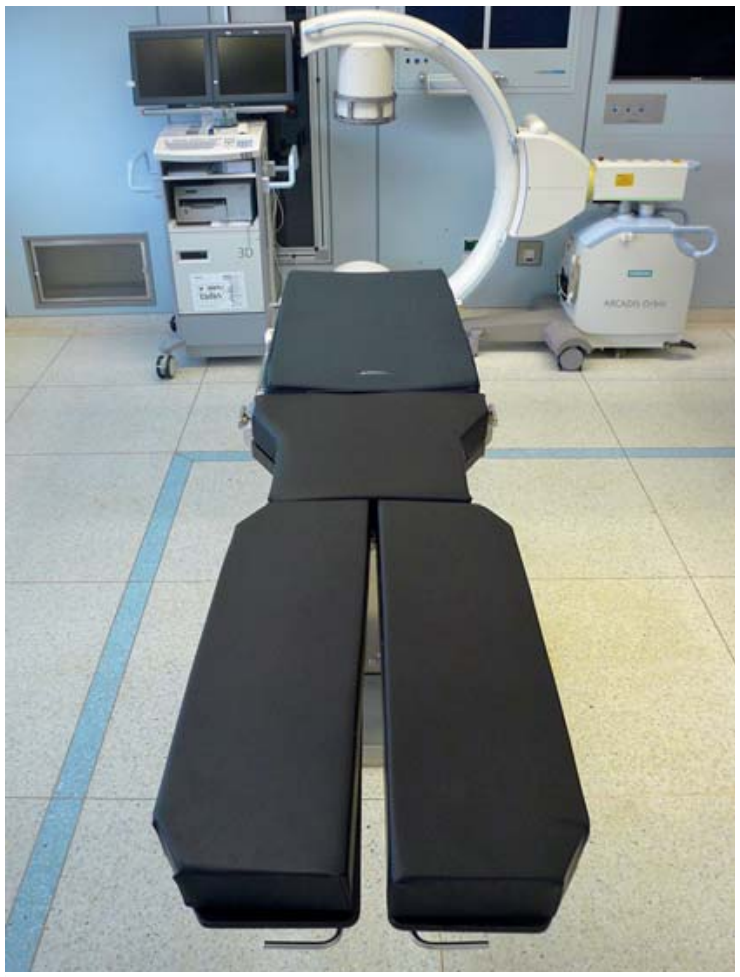

- Abb. 4 Sonder OP-Tisch „Becken“ 


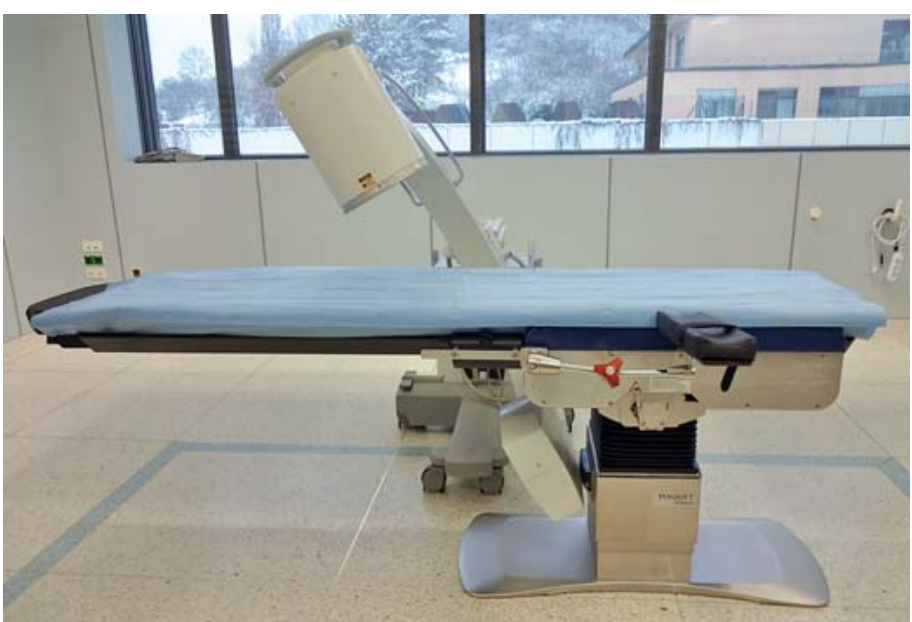

Abb. 5 OP-Tisch und C-Bogen

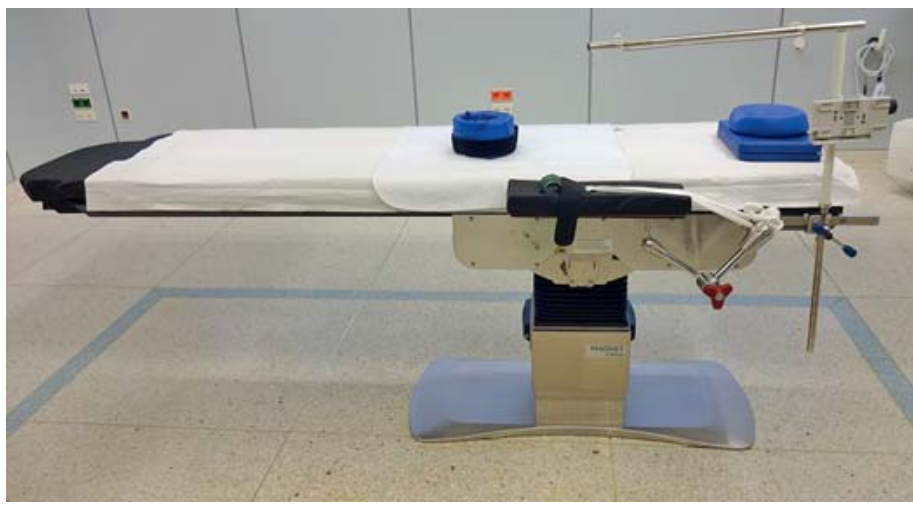

- Abb. 6 Carbon-OP-Tisch mit Auflagen

\section{Vorbereitung Lagerungshilfsmittel/ Lagerungsutensilien}

\section{Grundsätzlich}

- Rasierapparat (Clipper) zum Kürzen der Haare im OPGebiet

- Neutralelektrode mit Kabel

- Windeln zur Feuchtigkeitsprotektion

- durchsichtiges Pflaster

- Verbandsschere

- eventuell zusätzliche Moltontücher zum Abdecken des Patienten

\section{Speziell}

- Knierolle, Flockenkissen

- Tunnelkissen (bei Seitenlage ohne Vakuummatratze)

- eventuell Seitenstützen

- 2. Armlagerungsschiene „exzentrisch“ mit Kugelgelenk

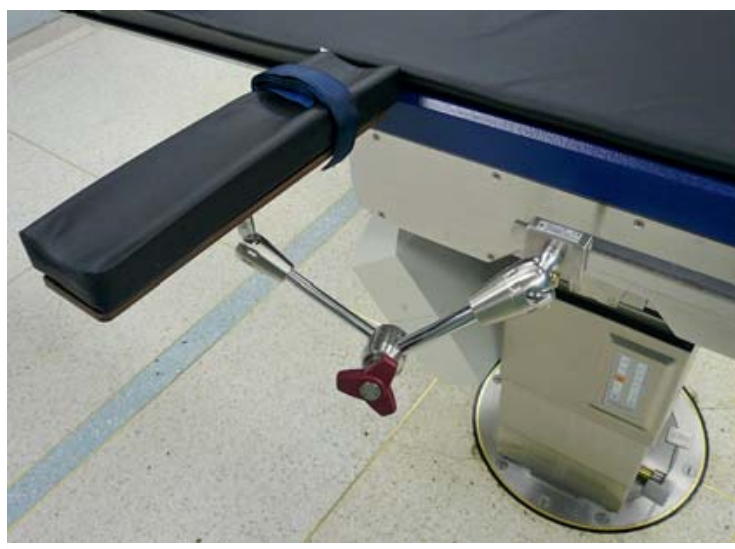

- Abb. 7 Armschiene mit Kugelgelenk

\section{Rasur}

Die Rasur des OP-Gebietes erfolgt nach modernen Hygienekriterien zeitnah zur Operation in der OP-Einleitung nach vorgegebenen Standards. Ein Kürzen der Haare mit einem elektrischen Clipper reicht nach modernen Hygienerichtlinien aus.

\section{Rückenlage}

Die Domäne der Rückenlagerung ist der vordere Beckenring, aber auch, je nach Frakturtyp, das Azetabulum und die ISG-Fugen.

\section{Klassische Zugänge}

- anteriorer Zugang zur Symphyse

- Stoppa, modifizierter Stoppa-Zugang

- ilioinguinaler Zugang/Zugang nach Letournel (bedingt)

- pararektaler Zugang

\section{Durchführung}

Nach Abschluss der anästhesiologischen Vorbereitung wird der Patient an das untere Ende des OP-Tisches verbracht. Hierfür verwenden wir unsere Inkontinenzunterlage, um möglichst Scherkräfte, v. a. bei älteren Patienten mit vorgeschädigter Haut, zu vermeiden.

Eine seitliche Ausrichtung/Verschiebung des Patienten auf dem OP-Tisch erfolgt ebenfalls mit der Safetex-Auflage. Dies ist abhängig davon, wo sich die „region of interest" befindet und ob ein intraoperativer 3-D-Scan oder ein CT geplant ist.

Die Beine werden, nach Bedeckung mit einem Moltontuch, mit einem Becken-Bein-Gurt fixiert. Oft ist es notwendig, ein Bein beweglich abzudecken. In diesem Fall wird nur ein Bein mit dem gepolsterten Beingurt fixiert. Dieser sollte zwar eng anliegen, aber nicht zu straff angezogen werden, um Druckschäden, v. a. Druck auf die Fersen, zu vermeiden. Von einer weiteren Unterpolsterung der Fersen und der Knie sehen wir routinemäßig ab. 


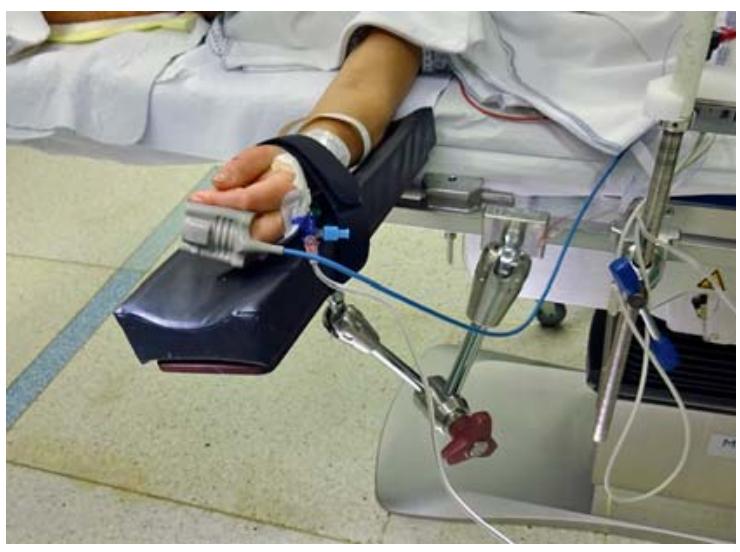

Abb. 8 Arm ausgelagert

Der Anästhesiearm kann auf der Armstütze mit Kugelgelenk „ausgelagert“ werden, hierbei ist darauf zu achten, dass diese bei den Durchleuchtungsmanövern nicht stört ( $\bullet$ Abb. 8). Andererseits ist sicherzustellen, dass es zu keiner Überstreckung im Plexus brachialis oder zur Druckschädigung durch OP-Tisch-Anbauten kommt (N. ulnaris).

Alternativ dazu kann der Anästhesiearm auch auf der exzentrischen Armschiene (Prototyp) über Kopf gelagert werden und der kontralaterale Arm, locker gesichert (Armfessel), auf der Brust abgelegt werden, sodass er bei der Inlet-Aufnahme nicht stört ( $\bullet$ Abb. 9).

Der Arm auf der Operationszugangsseite kann auch, am nach kranial geneigten Narkosebügel, mit Armfesseln gepolstert aufgehängt oder fixiert werden. Aber auch hier: "cave“ Metallkontakt. Das Ablegen des anderen Armes auf der Brust ist möglich, wie oben beschrieben.

Der OP-Tisch wird auf der Säule ganz nach fußwärts verschoben, um möglichst viel Platz für Inlet- und OutletDurchleuchtungseinstellungen zu haben. Auch die Durchführbarkeit von Ala- und Obturatoraufnahmen müssen sicher gewährleistet sein.

An den Flächen, an denen möglicherweise überschüssiges Desinfektionsmittel beim Desinfektionsvorgang ablaufen kann, schützen wir den Patienten vor dem „Nassliegen" durch Windeln, die wir so falten, dass sie eine optimale Saugwirkung entfalten. Nach dem Abwaschvorgang werden diese wieder entfernt ( $\bullet$ Abb. 10).

Manchmal kann es notwendig sein, ein kleines Lagerungskissen mittig unter das Becken zu positionieren. Dies ermöglicht eine zusätzliche ISG-Verschraubung gekoppelt mit ventralen Zugängen.

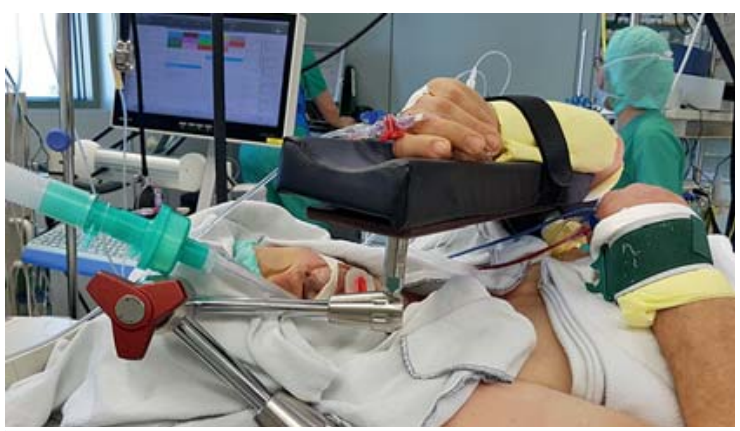

Abb. 9 Armlagerung

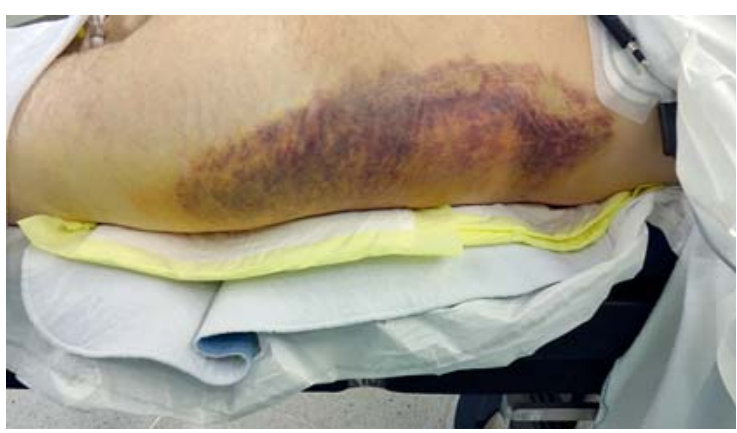

Abb. 10 Nässeschutz

\section{Wärmemanagement}

Während des gesamten Lagerungsvorgangs sollte darauf geachtet werden, dass der Patient immer wieder teilweise bedeckt wird, um ein Auskühlen möglichst zu verhindern. Hierfür stehen uns angewärmte Moltontücher aus der Narkoseeinleitung zur Verfügung. Die Anlage der Wärmemanagementsysteme der Anästhesie (z. B. Warmtouch) sollte, in enger Absprache, in den Lagerungsvorgang integriert werden und die Abwaschgrenzen respektieren. Das Inbetriebnehmen dieser sog. konvektiven Luftwärmer sollte aber, aufgrund möglicher Luftverwirbelungen, erst nach dem Abdeckvorgang erfolgen.

\section{Anlage der Elektrode für monopolaren Strom}

Wir haben uns in unserer OP-Abteilung für die Verwendung einer Neutralelektrode mit Äquipotenzialring entschlossen. Diese richten wir, obwohl es vom Hersteller nicht zwingend vorgeschrieben wird, immer standardmäßig zum OP-Gebiet aus, um den optimalen Stromfluss zu gewährleisten. Der Abstand der Neutralelektrode sollte so klein wie möglich zum OP-Gebiet sein, jedoch nicht weniger als $15 \mathrm{~cm}$ betragen. Ansonsten gelten die üblichen Anlagerichtlinien und die Herstellerangaben. Im Zweifelsfall empfehlen wir bipolare Stromanwendung. 


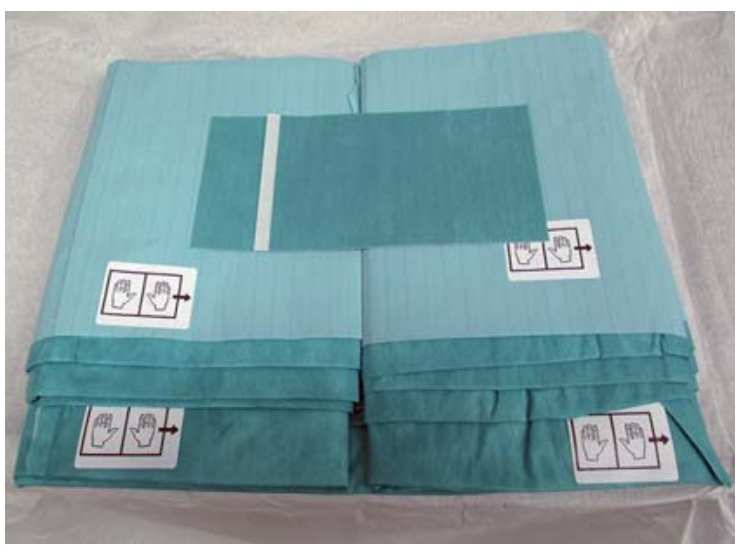

Abb. 11 „Schlitzsack“

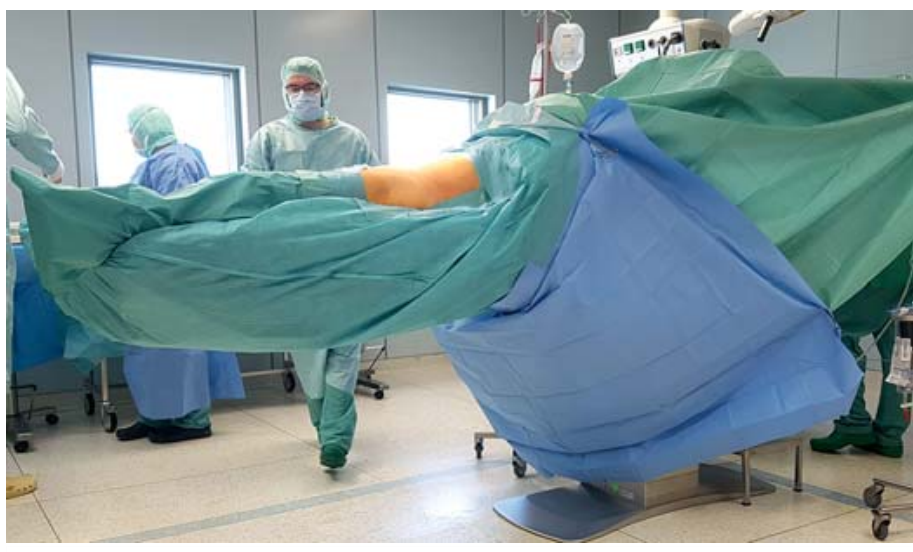

- Abb. 12 Abdeckung Becken

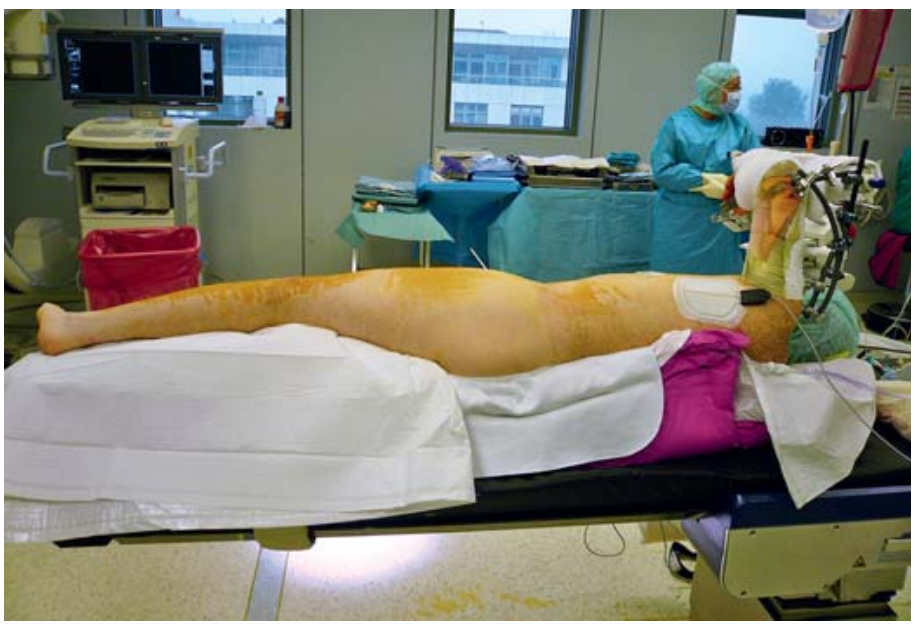

Abb. 14 Seitenlage Vakuummatratze

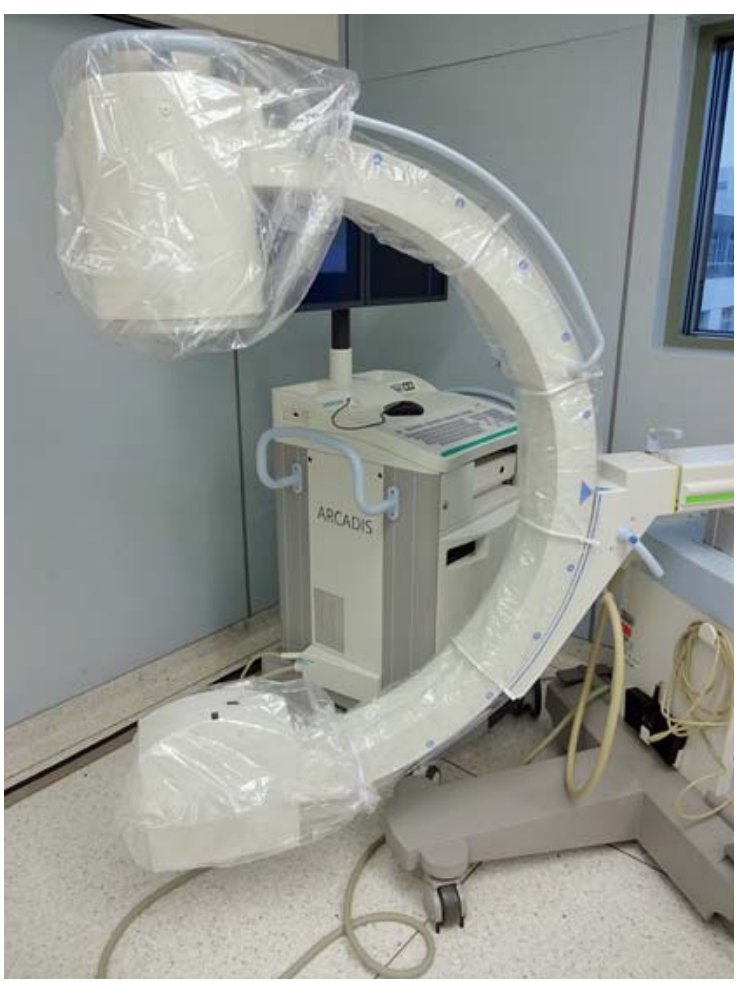

- Abb. 13 Abdeckung Bildverstärker

\section{Abdeckung und Bildverstärker}

Wir verwenden grundsätzlich eine U-Tuch-Abdeckung. Natürlich wäre auch in manchen Fällen eine Viereckabdeckung denkbar. Nach dem Desinfektionsvorgang wird das U-Tuch je nach Setup/Zugang anmodelliert und ein Quertuch angebracht. Wir verwenden zusätzlich einen für Tübingen speziell konzeptionierten Schlitzsack ( Abb. 11), der anschließend den gesamten OP-Tisch einpackt. Gegen die OP-Tisch-Säule wird noch ein „Back Table Cover“ oder sog. „Säulentuch“ gespannt. Dadurch ist der sich unter dem Patienten befindliche OP-Tisch bis zur Säule eingepackt ( $\bullet$ Abb. 12).

Der Bildverstärker wird mit einem sterilen Klammerbezug und je einem Überzug für Strahler und Detektor bezogen ( $\triangleright$ Abb. 13), somit kann während der Operation mehrfach durchgeschwenkt werden, ohne Verwendung von weiteren Schlepptüchern, Instrumentiertischbezügen oder Ähnlichem.

\section{Seitenlage}

Die Seitenlagerung kommt eher bei Versorgungen des Azetabulums über dorsale Zugänge zum Tragen.

\section{Klassische Zugänge}

- Kocher-Langenbeck

- Trochanterosteotomie (Digastric-trochanteric-Flip-Osteotomie) 


\section{Durchführung}

Als OP-Tisch verwenden wir denselben Carbon-OP-Tisch wie bei der Rückenlage. Es wird eine Vakuummatratze unter das Zelluloseabdecktuch gelegt. Es sollte ein leichtes Vorvakuum gezogen werden, sodass man die Matratze später beim Lagerungsvorgang gut anmodellieren kann ( $\triangleright$ Abb. 14).

Der Patient sollte möglichst am Ende der Vakuummatratze liegen und mit dieser an das Tischende verbracht werden. Nach dem Andrehen in Seitenlage wird die Vakuummatratze anmodelliert. Im OP-Bereich möglichst tief, um genügend Platz für den Zugang und die Abdeckung zu haben. Das Bein wird frei gelagert. Die Lagerung kann auf einem Tunnelkissen erfolgen. Es hat sich jedoch bewährt, das freie Bein nur mit Moltons zu unterpolstern. Der Patient wird mit Vakuummatratze und Becken-BeinGurt am OP-Tisch fixiert. Da intraoperative Seitkippungen des OP-Tisches notwendig werden können, hat es sich ebenfalls bewährt, im dorsalen Thoraxbereich eine Seitenstütze anzubringen. Der Narkosearm wird unter den gleichen Gesichtspunkten gelagert wie bei Rückenlage. Der freie Arm kann alternativ zur Armstütze mit Kugelgelenk in einer Göpel-Beinschale gelagert werden $(\triangleright$ Abb. 15). Es ist auch möglich, den Arm bequem quer zum ausgelagerten Anästhesiearm vor dem Gesicht auf einem Molton zu lagern.

Die Abdeckung erfolgt analog der Rückenlagerung mit einem U-Tuch. Auch in Seitenlage verwenden wir einen Schlitzsack und ein Säulentuch.

Die Vakuummatratze hat aus unserer Sicht mehrere große Vorteile:

- Der Druck wird gleichmäßig verteilt. Es entstehen dadurch weniger druckbedingte Lagerungsschäden.

- Die Vakuummatratze hat zumindest in der Theorie eine isolierende Wirkung und unterstützt somit das Wärmemanagement.

- Bei richtiger Anwendung bleibt die Lagerung bis zum OP-Ende unverändert.

- Die Durchleuchtung wird durch die Vakuummatratze nicht beeinflusst.

\section{Bauchlage}

Ermöglicht Zugänge zum Sakrum, den ISG-Fugen und den dorsalen Beckenanteilen.

\section{Durchführung}

Auch hier hat sich der Becken-Carbontisch bewährt.

Wir drehen den Patienten mit einer besonderen kinästhetischen Technik ohne besonderen Kraftaufwand mit einem 2. "Safetex“ auf dem OP-Tisch auf den Bauch. Dadurch ersparen wir uns einen 2. OP-Tisch und die damit verbundenen Arbeiten. Auch bei der Bauchlagerung wird

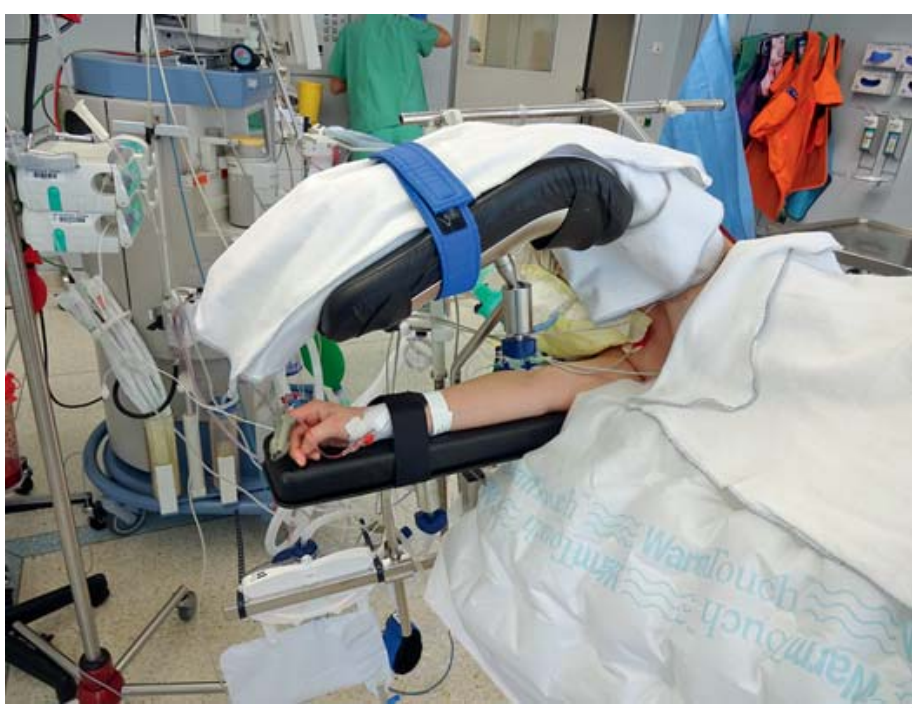

Abb. 15 Armlagerung Seitenlage

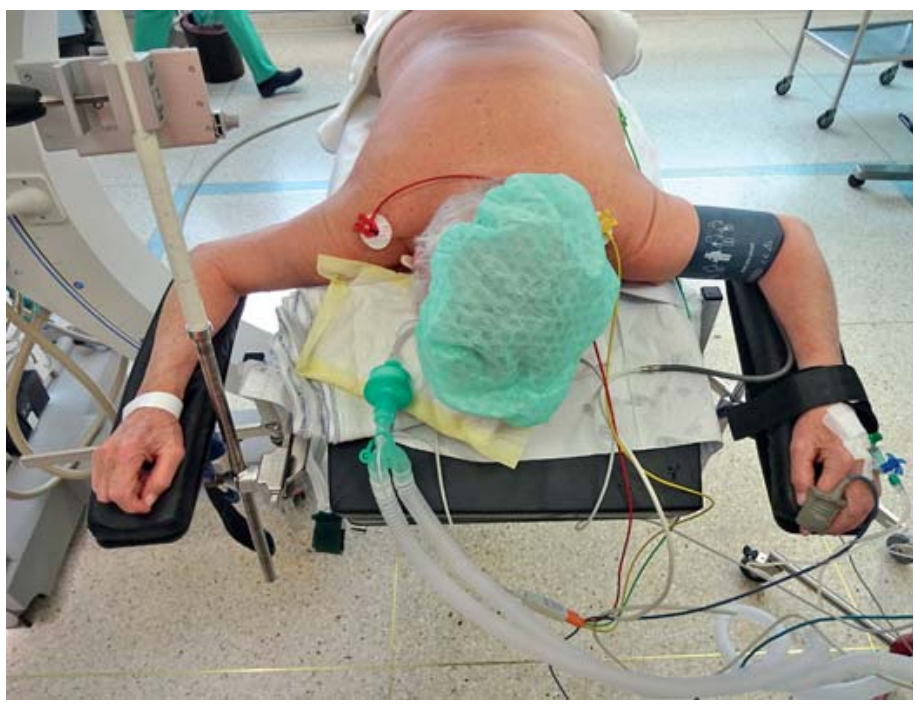

Abb. 16 Armlagerung Bauchlage

der Patient an das Fußende des OP-Tisches gelagert. Die Unterpolsterung des Thorax (Beatmung) und des Beckens (Entlastung der Beckengefäße) gestalten wir eher „puristisch“, fraktur- und patientenadaptiert und damit sehr Individuell. Wir verwenden, wenn überhaupt, zusammengerollte Moltons unter Verwendung der Polsterung der Weichschaumlagerungsmatratze des OP-Tisches. Industriell vorgefertigte Lagerungskissen kommen bei uns nur selten bis gar nicht zur Anwendung. In letzter Zeit kommen hier jedoch vermehrt „Flockenkissen“ zum Einsatz! Bei der Lagerung des Kopfes haben wir sehr gute Erfahrungen mit der direkten Lagerung zur Seite auf dem OP-Tisch oder mit einem Tempur ${ }^{\circledR}$-Kopfring gemacht. Zusätzlich bringt diese Art der Lagerung eine höhere Stabilität mit sich, die bei der Osteosynthese von großem 
Vorteil ist. Eine Knierolle sorgt für Entlastung der Zehen und der Gefäß-Nerven-Bündel im Kniegelenk. Die Arme lassen sich sehr schön in entspannter 90/90-Lagerung unter Entlastung des Plexus brachialis auf Armschienen auslagern. Auch hier haben sich die Armschienen mit Kugelgelenk bewährt ( $\triangleright$ Abb. 16). Auch eine Lagerung in einer Vakuummatratze wäre denkbar.

Die Abdeckung erfolgt analog der Rücken- und Seitlagerung, auch hier kann man ein oder 2 Beine mitabdecken, was nur selten notwendig ist.

\section{Zusammenfassung}

Die Lagerungen zur Versorgung von Beckenfrakturen sind ein komplexes Ereignis, v. a. besonders aufgrund der meist assoziierten Kollateralverletzungen. Hier ist ein hohes Maß an Absprache (Teamwork) sowie ein hohes Maß an Erfahrung notwendig. Standards sind äußerst hilfreich, müssen jedoch immer wieder patienten- und frakturbedingt oder aufgrund des operativen Vorgehens erweitert oder abgeändert werden.

Interessenkonflikt

Die Autoren erklären, dass kein Interessenkonflikt vorliegt.
Autorinnen/Autoren

\section{Alexander Motzny}

Str. OP-Leitung, Leiter AEMP, BG Klinik Tübingen

Tobias Maximilian Kraus

Priv-Doz. Dr., BG Klinik Tübingen

Armin Mees

Fachkrankenpfleger OP, BG Klinik Tübingen

Korrespondenzadresse

\author{
Alexander Motzny \\ BG Klinik Tübingen \\ Schnarrenbergstraße 95 \\ 72076 Tübingen \\ amotzny@bgu-tuebingen.de
}

\section{Bibliografie}

DOI https://doi.org/10.1055/a-0585-0971

OP-JOURNAL 2018; 34: 54-60 @ Georg Thieme Verlag KG Stuttgart · New York ISSN 0178-1715 